\title{
Aus den Verhandlungen des FMH-Zentralvorstands
}

\author{
La version française
} suivra

\begin{abstract}
St. An seiner Sitzung vom 24. Februar 2005 behandelte der FMH-Zentralvorstand unter anderem folgende Geschäfte.
\end{abstract}

\section{Strukturreform}

An die Mandatsträger der in der Ärztekammer vertretenen Organisationen und Gremien wurden rund 800 Fragebogen zur Strukturreform verschickt. Erwartet wird ein Rücklauf von mindestens $40 \%$. Einige Personen haben den Fragebogen anonym zurückgeschickt; dies hat zur Folge, dass auch diese Personen Mahnung(en) erhalten werden.

Weitere Sitzungen des Leitungsgremiums haben stattgefunden. Eine kleine Gruppe, bestehend aus J. de Haller, A. Müller Imboden und Hr. Bürki von der Beratergruppe für Verbandsmanagement (B'VM), welche die Reform begleitet, wird die Koordination zwischen den Strategieüberlegungen des Zentralvorstandes und den strukturellen Projekten des Leitungsgremiums übernehmen.

Es gibt Interessenten für den Fragebogen, die nicht in der Liste der Chargierten der FMH sind und den Fragebogen also nicht erhalten haben. Sie können aber ihre Meinungen und Anregungen individuell erfassen und an die B'VM kommunizieren.

Die Änderungen der FMH-Statuten soll in diesem Herbst erfolgen; die Inkraftsetzung ist per 1. Januar 2006 vorgesehen.

\section{Delegierte Psychotherapie: Rückwirkende Inkraftsetzung}

Das Fähigkeitsprogramm «Delegierte Psychotherapie» ist von den zuständigen Gremien - Kommission für Weiter- und Fortbildung (KWFB), Präsidentenkonferenz, Ärztekammer - verabschiedet worden. Der Zentralvorstand setzt es rückwirkend per 1. Januar 2005 in Kraft und genehmigt gleichzeitig den zugehörigen Vertrag mit der Foederatio Medicorum Psychiatricorum et Psychotherapeuticorum (FMPP), dem Zusammenschluss der Schweizerischen Gesellschaften für Psychiatrie und Psychotherapie sowie für Kinder- und Jugendpsychiatrie und -psychotherapie.

\section{3. «Tauchmedizin» im Telefonbuch: Vereinbarung mit der Swisscom}

Die Schweizerische Gesellschaft für Unterwasserund Hyperbarmedizin hat ein Gesuch um Aufnahme eines Rubriktitels «Tauchmedizin» in den schweizerischen Telefonbüchern gestellt. Das KWFB-Büro hat anlässlich seiner Sitzung vom 3. Februar 2005 dieses Ansinnen gutgeheissen und der Ergänzung des Rubriktitels «Tauchmedizin» in der Vereinbarung mit der Swisscom zugestimmt.

\section{Weiterbildungsausschuss: Wechsel im Präsidium}

Das Bundesamt für Gesundheit (BAG) hat der FMH am 22. Dezember 2004 mitgeteilt, dass Prof. U. Lütolf, Zürich, die Nachfolge von Dr. R. Salzberg als Präsident des Weiterbildungsausschusses angetreten hat. Dieser Ausschuss beurteilt die Gesuche um Anerkennung eines Weiterbildungstitels für medizinische Berufe. Der Zentralvorstand nimmt das Schreiben des BAG zur Kenntnis, verdankt das Engagement von Dr. Salzberg und gratuliert Prof. Lütolf zur Wahl.

\section{Richtlinien Ärzteschaft und Industrie}

Die Schweizerische Akademie der Medizinischen Wissenschaften (SAMW) hat unter Mitarbeit der FMH Richtlinien zu diesem Thema erarbeitet, welche die Empfehlungen von 2002 ersetzen sollen. Der Zentralvorstand ist sich einig, dass es in diesem Bereich Richtlinien braucht. Der Entwurf ist jedoch nicht reif für eine definitive Publikation. Das Projekt soll nach Diskussion in der Präsidentenkonferenz vom 17. März 2005 an die SAMW zur Überarbeitung zurückgehen und die aktuelle Fassung so überarbeitet werden, dass der Zentralvorstand dahinterstehen kann.

\section{Weiterentwicklung EPA-Projekt}

Die von der FMH unterstützte EPA-Pilotstudie (European Practice Assessment) wurde erfolgreich durchgeführt und abgeschlossen. Hinter- 
grund und erste Ergebnisse sind bereits publiziert (Verlag Bertelsmann-Stiftung).

Die Implementierung des EPA-Instruments wird in den neun teilnehmenden Ländern unterschiedlich erfolgen. In der Schweiz hat die FMH mit der Übernahme der Lizenzgebühren für ein webbasiertes Feedbacksystem den Grundstein gelegt, um aus einem zeitlich befristeten Forschungsprojekt eine nachhaltige Dienstleistung für die Qualitätsförderung in der ambulanten Medizin zu entwickeln. Damit diese Entwicklung zu einem Qualitätsförderungsprogramm möglichst breit abgestützt werden kann, muss das EPA-Instrument um klinische Indikatoren erweitert werden. Deshalb wird ein Entwicklungskredit in der Höhe von Fr. 45 000.- für das laufende Jahr - budgetiert. Der Zentralvorstand genehmigt diesen Kredit.

\section{Projekt Morbiditätsindikatoren}

Geplant ist die Durchführung eines mehrteiligen Projektes mit der Absicht, ein schweizweit einheitliches System zur Dokumentation der Morbiditäten der versorgten Patientenpopulation zu entwickeln bzw. zu adaptieren und zum Einsatz zu bringen. Ziel des Projektes ist es, ein FMHMorbiditätsobservatorium aufzubauen, d.h. die Entwicklung bzw. Unterstützung von Instrumenten zur Schaffung von Transparenz bezüglich Strukturen und Prozessen in der medizinischen Versorgung in der Schweiz. Je breiter das Projekt abgestützt ist, desto grösser sind die Erfolgschancen.

Das Projekt wird in vier Phasen aufgeteilt: Vorstudie, Detailstudie, Pilotphase und Einführung. Die Personalressourcen müssen im GS vorhanden sein, ansonsten müssen diese auswärts eingekauft werden. Die geschätzten Kosten belaufen sich auf total Fr. 592 000.-.

Der Zentralvorstand unterstützt dieses Projekt; es wird an der Präsidentenkonferenz vorgestellt.

\section{Projekt Démographie Médicale}

Die FMH wurde vom «Observatoire suisse de la santé» (OBSAN) bezüglich einer allfälligen Kooperation im Projekt Ärztedemographie angefragt. Konkret will das OBSAN einen FMH-Vertreter in der Arbeitsgruppe und einen FMHVertreter im Steuerungsausschuss.

Bis jetzt sind folgende Organisationen im Steuerungsausschuss vertreten: Bundesamt für Statistik, Bundesamt für Gesundheit, Gesundheitsdirektorenkonferenz sowie das OBSAN. Eine Vertretung der FMH in diesem Ausschuss wird vom ZV als sinnvoll erachtet; der ZV mandatiert L. T. Heuss. G. von Below und J. Jau werden in der Arbeitsgruppe mitarbeiten. Finanziell wird sich die FMH nicht am Projekt beteiligen.

\section{Arbeitssicherheit in der Arztpraxis: Rolle der FMH}

Die revidierten gesetzlichen Grundlagen für den Gesundheitsschutz am Arbeitsplatz sind schon seit Jahren in Kraft. Alle Arbeitgeber mit mehr als fünf Mitarbeitenden müssen nach den Richtlinien der Eidgenössischen Kommission für Arbeitssicherheit (EKAS) eine formelle Risikoanalyse durchführen. Jeder Arbeitgeber hat die gesetzliche Pflicht, den Arbeitnehmerschutz sicherzustellen. Auch Klein- und Kleinstbetriebe wie Einzelpraxen müssen sicher sein; allerdings sind bei ihnen die Anforderungen geringer. Eine auf die konkrete Situation der Arztpraxis ausgelegte Checkliste könnte helfen, mit möglichst wenig Bürokratie einen möglichst guten Gesundheitsschutz am Arbeitsplatz zu erreichen. Das Ressort Prävention/Public Health im Generalsekretariat wird die Schweizerische Gesellschaft für Arbeitsmedizin SGARM kontaktieren und bei Vorliegen eines konkreten Projektes den Zentralvorstand informieren. 